\title{
VALUE OF THE COMBINED TEST IN PRENATAL DIAGNOSTICS
}

\author{
Lončar D
}

*Corresponding Author: Dragan Lončar, Gynecology and Obstetrics Clinic, Clinical Center Kragujevac , Vojislava Kalanovića 1A/3, 34000 Kragujevac, Serbia; Tel.: +381-64-616-8999; E-mail: drloncar@sezampro.rs

\begin{abstract}
Congenital anomalies are the cause of 20.0$25.0 \%$ of cases of perinatal death, while $3.0 \%$ of children are born with malformations of varying size. We examined the predictive values and defined the credibility ratio of the combined test results. Of 317 examined pregnant women, $16(5.05 \%)$ gave a pathological karyotype after amniocentesis: of these, nine $(2.84 \%)$ had chromosomal number aberrations and seven $(2.21 \%)$ had chromosomal structure aberrations. We determined the ultrasonographic parameters using the standards of the Fetal Medicine Foundation (location please). We measured free $\beta$-subunit of choriogronadotropin ( $\beta$-HCG) and pregnancy associated plasma protein A (PAPP-A) in venous blood from pregnant women using a combined commercial assay. Sensitivity of the test is $94.0 \%$, and specificity is $99.0 \%$. The positive likelihood ratio [likelihood ratio test $(\mathrm{LR}+)$ ] is 94.00 , a negative likelihood ratio is [likelihood ratio test (LR-)] 0.06. The pretest probability that pregnant women carry a fetus with chromosomal abnormality is $1: 250$. Posttest odds after the combined test to discover this abnormality is 0.3760 , and probability of the same case is 0.2732 if it happens that the test
\end{abstract}

Gynecology and Obstetrics Clinic, Clinical Center Kragujevac, Kragujevac, Serbia result is positive. The result of our study confirms the justification of combined test usage in routine clinical practice, since the posttest odds rate in the case of a positive screening increases several times over (almost 90 times); the probability of detecting a chromosomal abnormality was about 70 times. The combined screening test, if used methodologically correctly, has a high predictive value in detecting fetal congenital anomalies.

Key words: Predictive value; Combined test; Ultrasonography; Biochemical markers

\section{INTRODUCTION}

Congenital anomalies cause $20.0-25.0 \%$ of perinatal deaths, while $3.0 \%$ of children are born with malformations of varying size [1]. Analytical immunochemiluminescence assays and an automated analyzer IMMULITE 2000 [Diagnostics Product Corporation (DPC), Los Angeles, CA, USA] were used. Usable values of the combined test were estimated on the basis of its sensitivity, specificity and possibility of the disease in case the result was positive. By combining the values of pregnancy-associated plasma protein A (PAPP-A) and free $\beta$-subunit of choriogonadotropin (free $\beta$-HCG) in serum with nuchal translucency (NT) diameter (combined test), the possibility of detecting trisomy 21 rises up to $90.0 \%$ with $5.0 \%$ false-positive findings [2]. The testing was 
done between 11 and $13^{+6}$ weeks of gestation. If the result happened to be positive, some invasive methods of prenatal diagnosis were suggested to the pregnant woman. A limit value of the combined test was 1: 250. A special problem was the test result interpretation. According to the literature, $32.0 \%$ of pregnant women did not know what the term "high risk" meant after getting the results and talking to the doctor [3]. The research objective was: 1) to examine the sensitivity and specificity of ultrasonographic (NT) and biochemical (free $\beta$-HCG and PAPP-A) markers as parameters of the combined test, and amniocentesis in diagnostics of congenital fetal anomalies; and 2) to set the credibility ratio of the combined test results.

\section{MATERIALS AND METHODS}

The prospective, observational study was conducted at the Gynecology and Obstetrics Clinic at the Clinical Center Kragujevac (GOC, CC Kragujevac, Kragujevac, Serbia) during 2008 and 2009 on singleton intrauterine pregnancies in the first trimester of pregnancy. The clinical and experimental model of study was used throughout the research. The Ethics Committee at the CC Kragujevac confirmed the correctness of this study and authorized its conduct. Three hundred and seventeen pregnant women were included in the examination and observed by the Board of Genetic Counseling at GOC, CC Kragujevac. An informed consent form was signed by all participants. All ultrasound examinations were conducted on the Aloka Prosound 3500 Ultrasound Machine Package with UST-9124 (Aloka, Seoul, South Korea) at GOC, CC Kragujevac. Pregnancy was $11-13^{+6}$ weeks of gestation. The crown-rump length (CRL) of the fetus was between 45 and $84 \mathrm{~mm}$. While measuring fetal nuchal translucency (NT), we used the ultrasound apparatus of high resolution with the option"cine loop" so that the image could be turned by calipers that allow measurement of one decimal point. The screen image on which NT was measured, encompassed only the head and the upper part of fetal rib cage. We used the maximum enlargement, so that small movements of the caliper altered the diameter by only $0.1 \mathrm{~mm}$. The NT was measured by transvaginal approach of color Doppler technique with the fetus in a neutral position. We measured the maximal thickness of subcutaneous illumination between the skin and soft tissue located above the cervical part of the fetal spine. We set the calipers on lines that define the fold, so that they were barely visible on the white limit line of the accumulation behind the neck. During the examinations we conducted more measurements and took into consideration only the highest thickness of the NT. We carried out the quantitative settings of free $\beta$-HCG and PAPP-A from venous blood of patients by applying commercial tests from DPC. Tests were based on the analytical immunochemiluminescence assay and were realized by using the automated analyzer IMMULITE 2000 (DPC).

All the pregnant women included in this study underwent amniocentesis by a standard transabdominal procedure with the ultrasound control using the "free hand" technique at 16 to $17^{+6}$ weeks of gestation. We performed the amniocentesis by needles with mandrene of $20-22 \mathrm{G}$ thickness and aspirated $15-20 \mathrm{~mL}$ of amniotic fluid into a syringe without a rubber seal. The amniotic fluid sample was delivered to the Cytogenetics Laboratory at GOC, CC Kragujevac, and all results received were deposited into the unique database with required logistic control.

\section{RESULTS}

After conducting the combined test in the total sample of pregnant women, we found the following individual values of the examined parameters (see Table 1): the statistically significant difference in values of free $\beta-\mathrm{HCG}$ and NT in the examined group of pregnant women was $p<0.05$. Parameter PAPP-A does not show any statistically significant difference in the examined group of pregnant women. We also found the same characteristics of the examined parameters in the receiver operating characteristic (ROC) curve analyses in the examination of predictive characteristics of the specified parameters (Figures 1 and 2).

Analysis of the value distribution of the NT thickness measurement showed that the distribution was regular and that measurements were being set regularly around the median $(44.0 \%$ below and $56.0 \%$ above median), which was in accordance with the criteria for quality control established by 


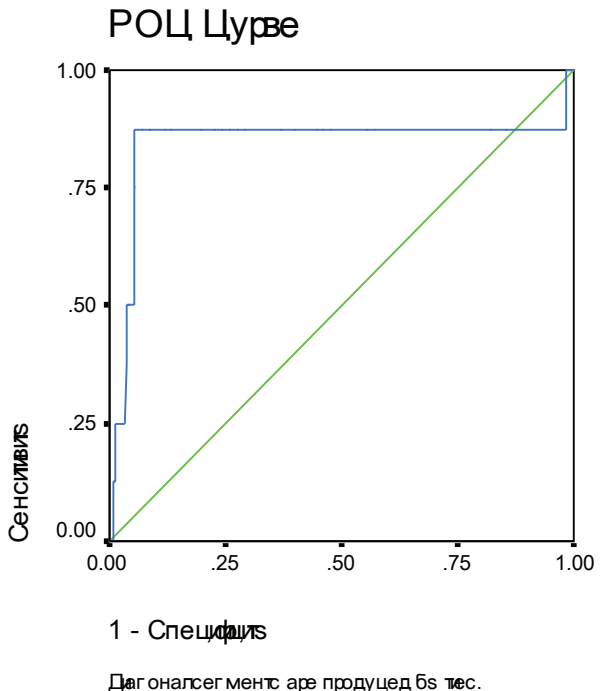

Figure 1. Probability of the predictive value of free fraction $\beta$-HCG [field below ROC curve $0.846,95 \%$ confidence interval (95\% CI) 0.628-1.064].

the Fetal Medicine Foundation (London, UK) and was supposed to be $40.0-60.0 \%$ above the median. The distribution of fetal NT for given CRL in examination was no different from the established distribution of the Fetal Medicine Foundation used as a standard. On the basis of that, our measurements of NT thickness can be considered to be regularly conducted and usable in further examination.

The diameter of NT did significantly statistically differ in the examined group of pregnant women $(p<0.05)$. Crown-rump length and gestational age were not different statistically $(p>0.05)$. Using the contingency table (Table 2), we set the predictive value of the combination of ultrasonographic and biochemical markers after taking over the results of amniocentesis.

Estimation of probability that some disease is present before testing is called pretest probability ("a priori probability"). Pretest probability is received on the basis of available information about the patient, also including testing previous to the actual one. Estimation of the probability of disease after testing is called posttest probability ("a posteriori probability"). Posttest probability is less or higher than pretest probability depending on the test results. Measures of diagnostic accuracy (sensitivity, specificity) cannot directly answer the following important clinical questions: 1 ) if the disease pretest

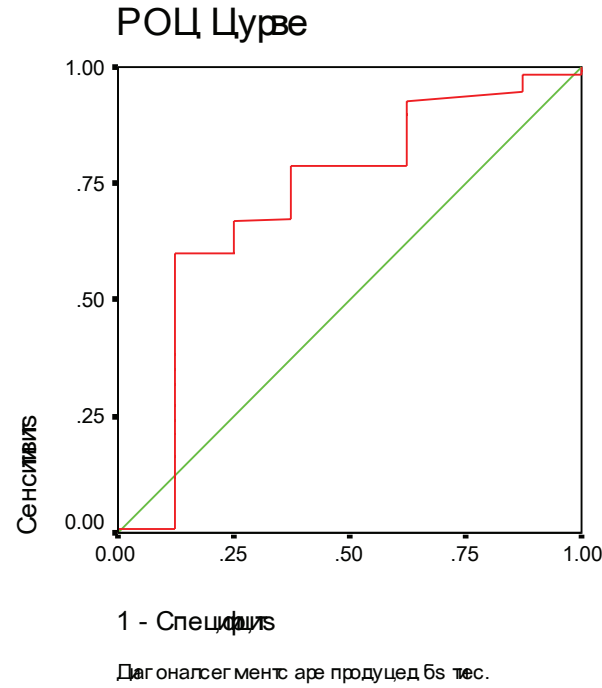

Figure 2. Probability of the predictive value of PAPP-A [field below ROC curve 0.715 (95\% CI 0.508-0.921)].

probability is known, and the examinee is positive on the test, what is the probability that he/she really has the disease? 2) If the disease pretest probability is known, and the examinee is negative on the test, what is the probability that he/she really does not have the disease?

These questions can be answered by application of the pretest odds of the disease and the credibility ratio. Disease odds ratio is the ratio of probability that the disease is present $(p)$ and probability that is not present $(1-p)$ : odds $=p / 1-p$. According to that, pretest disease odds are: pretest odds $=$ pretest probability/1-pretest probability. Likelihood ratio (LR) is the probability ratio of the certain test result $(+$ or - ) of the examinee who has the disease divided with the probability of the same result of the person who does not have the disease. Two types of likelihood can be calculated: 1) likelihood ratio of the positive test ( $\mathrm{LR}+)$ is the ratio of sensitivity and false positive ratio (1-specificity): LR $+=$ sensitivity/1-specificity; 2) likelihood ratio of the negative test (LR-) the ratio of sensitivity and false negative ratio (1sensitivity) and specificity: LR $-=1$-sensitivity/ specificity. The likelihood ratio shows how the test result can alter the pretest disease probability. The LR+ shows how much the test result increases disease probability, LR- shows how much the test 
Table 1 Difference between values of free $\beta$-HCG, PAPPA and NT in 317 pregnant women (total sample).

\begin{tabular}{|l|c|c|c|}
\hline Parameters & Free $\boldsymbol{\beta}$-HCG $(\mathbf{m L} / \mathbf{m L})$ & PAPP-A $(\mathbf{m L} / \mathbf{m L})$ & NT (mm) \\
\hline Pathological karyotype & 114.00 & 1.36 & 2.55 \\
\hline Physiological karyotype & 19.20 & 1.84 & 1.90 \\
\hline Mann-Whitney test $(\mathrm{U})$ & 704.50 & 2191.00 & 621.50 \\
\hline$p$ Value & 0.000 & 0.543 & 0.000 \\
\hline
\end{tabular}

Table 2. Review of the middle values (MV) and standard deviations (SD) of the ultrasonographic parameters and gestational age in 317 pregnant women

\begin{tabular}{|l|c|c|c|}
\hline Parameters & $\begin{array}{c}\text { Pathological Karyotype: } \\
\mathbf{1 6}\end{array}$ & $\begin{array}{c}\text { Control Group: } \\
\mathbf{3 0 1}\end{array}$ & p Value \\
\hline Nuchal translucency (mm) & $2.49 \pm 0.37$ & $1.92 \pm 0.39$ & $<0.05$ \\
\hline Crown-rump-length (mm) & $60.12 \pm 8.48$ & $64.83 \pm 8.23$ & $>0.05$ \\
\hline Gestational age (days) & $86.69 \pm 3.98$ & $87.40 \pm 7.40$ & $>0.05$ \\
\hline
\end{tabular}

result decreases disease probability. The likelihood ratios are not under the influence of the disease prevalence.

Likelihood ratios can help measuring the posttest probability. How big the change from pretest to posttest probability depends considerably on the values of the likelihood ratio. It is desirable for $(\mathrm{LR}+)$ to have the highest values and (LR-) to have values closest to 0 . For calculating the posttest disease probability, posttest odds are first to be calculated. 1) For positive test result: posttest odds $=$ pretest odds $\times \mathrm{LR}+$; 2) for negative test result: posttest odds $=$ pretest odds $\times$ LR - . Posttest probability is obtained by the formula: posttest probability $=$ posttest odds $/ 1+$ posttest odds. According to the literature data, the diagnostic accuracy of the combined test, in relation to the result of the early amniocentesis (referral standard) is: sensitivity 0.88 , specificity 0.90 . In our sample sensitivity is 0.94 and specificity 0.99 . Likelihood ratios: LR+ $=0.94 / 1-0.99=94.00 ;$ LR $-=1-0.94 / 0.99=0.06$. Pretest probability that the pregnant woman carries the fetus with the chromosomal abnormality is $1: 250=0.004$. Pretest odds $=0.004 / 0.996=0.004$. If the test is positive: posttest odds $=$ pretest odds $\times$ $\mathrm{LR}+=0.004 \times 94=0.3760 ;$ posttest probability $=$ posttest odds $/ 1+$ posttest odds $=0.3760 / 1+0.3760$ $=0.2732$. If the test is negative: posttest odds $=$ pretest odds $\times$ LR $-=0.004 \times 0.06=0.00024$. Posttest probability $=$ posttest odds $/ 1+$ posttest odds $=0.00024 / 1+0.00024=0.00024$.

\section{DISCUSSION}

In our study, we had nine $(2.84 \%)$ pregnant women with numeric aberrations in total and seven (2.21\%) pregnant women with structural aberrations in fetuses which could be explained by the fact that the sample was preselected. All pregnant women were sent to Genetic Counseling at GOC, CC Kragujevac (Table 2), for some suspicious reason (positive personal and/or family case history, age of the pregnant woman, giving birth to child with chromosomal aberrations and/or fetal anomalies in previous pregnancies, etc.). Similar results were reported in the study conducted in Great Britain in 2000, stating that the total incidence of Down's syndrome 2, 1 in 1,000 deliveries, which was 50.0\% more than in the national reports [4].

The importance of the NT measurement in screening for Down's syndrome during the first trimester of the pregnancy was recognized back in 1990. With the limit value of $3 \mathrm{~mm}$ NT thickness, the detection rate (DR) is $64.0 \%[5,6]$. Screening sensitivity of chromosomopathies in comparison to NT was $75.0 \%$ with the value of false-positive ratio of $2.1 \%$ [6]. In our sample, 11 pregnant women in total from the group of 16 had a measured value of NT above the median for the given CRL in the group of pathological karyotypes that was $68,75.0 \%$. By the analysis of the total sample, we found that with 26 pregnant women we measured a NT of $2.55 \mathrm{~mm}$ above median for the given CRL and by invasive 
diagnostics we confirm 16 cases of chromosomal fetal aberrations or $61.54 \%$ (Table 3). Methodology of the combined test (Table 1) indicated that the ultrasound screening was done first, and after that to set the level of free $\beta-\mathrm{HCG}$ and PAPP-A, where risks are calculated as the combination of the two data [7]. For a certain gestation time, the levels of free $\beta$-HCG and PAPP-A represent the factor of probability, which is multiplied by the initial risk in order to calculate the new risk [8]. Differences in the concentration of free $\beta$-HCG between normal pregnancies and those with trisomy 21 is increasing, and differences in the level of PAPP-A is decreasing with the length of the pregnancy.

There is no significant connection between thicknesses of the fetal NT, level of free $\beta$-HCG or PAPP-A in maternal serum in pregnancies with trisomy 21 in relation to normal pregnancies, so ultrasound and biochemical markers can be combined in order to get more efficient screening results. Numerous studies have confirmed the connection between the low level of PAPP-A and trisomy 21 during the first trimester [2]. In normal pregnancies, the level of PAPP-A in maternal blood increases with gestation time, and in pregnancies with trisomy 21 it decreases [multiple of median $(\mathrm{MoM})](<0.5)$. By setting the value of PAPP-A, it is possible to detect $52.0 \%$ of Down's syndrome cases with $5.0 \%$ falsepositive results [2]. In pregnancies with trisomy 21 , the level of free $\beta-\mathrm{HCG}$ is increased between 8 and 14 weeks of gestation. The level of free $\beta$-HCG in maternal blood decreases normally with gestation time, and in pregnancies with trisomy 21 , the level of free $\beta-H C G$ increases $(\mathrm{MoM}>2.0)[9,10]$. On the basis of free $\beta$-HCG level, DR amounts to $42.0 \%$ with $5.0 \%$ false-positive findings [11]. Frequency of false-positive results, according to the available literature, is estimated at 5.0\% [12-15].

Our research has shown that the rate of the false-positive findings is $1.0 \%$, and that free $\beta$-HCG is a more sensitive predictor than PAPP-A. Other investigators, have reported identical conclusions [14]. Predictive value of the individual biochemical markers is represented at charts 1 and 2 by setting the area below the ROC curve. On reviewing the combined test predictive value of our sample of pregnant women, we found the following results: sensitivity of the test is $94.0 \%$, specificity is $99.0 \%$.

Table 3. Contingency table created on the basis of data processing in the total sample of pregnant women after amniocentesis

\begin{tabular}{|l|c|c|c|}
\hline Test Result & Disease Present & Disease Absent & Total \\
\hline Positive & Really positive: 15 & False-positive: 1 & RP+FP: 16 \\
\hline Negative & False-negative: 3 & Really negative: 298 & FN+RN: 301 \\
\hline Total & 18 & 299 & 317 \\
\hline
\end{tabular}

Table 4. Probability and predictive values of the parameters of the combined test in relation to result of early amniocentesis

\begin{tabular}{|l|c|c|}
\hline Parameter & p Value & $\mathbf{9 5 \% ~ C I ~}$ \\
\hline Sensitivity & 0.9375 & $0.6977-0.9984$ \\
\hline Specificity & 0.9900 & $0.9712-0.9979$ \\
\hline Positive predictive value & 0.8333 & $0.5858-0.9642$ \\
\hline Negative predictive value & 0.9967 & $0.9815-0.9999$ \\
\hline Prevalence & 0.0505 & $0.0291-0.0807$ \\
\hline False-positive rate & 0.0100 & $0.0046-0.0245$ \\
\hline False-negative rate & 0.0625 & $0.0145-0.0998$ \\
\hline Positive likelihood ratio (LR+) & 94.0000 & $30.2937-292.0653$ \\
\hline Negative likelihood ratio (LR-) & 0.0631 & $0.01746-1.2712$ \\
\hline Overall test accuracy & $\mathbf{0 . 9 8 7 3}$ & $0.07215-0.9981$ \\
\hline
\end{tabular}


The positive predictive value of the test is 0.83 , and negative predictive value of the test is $0.99(99.0 \%)$. The positive likelihood ratio ( $\mathrm{LR}+)$ is 94.00 and negative likelihood ratio (LR-) is 0.06 (Tables 4 and 5). We have already confirmed the published positive qualifications of this screening method $[11,16]$ and point to its justification in every day clinical practice [17] regarding posttest odds rate in case of positive screening increases several times over (almost 90 times). In the available literature, we have not found any reports that have the calculation of the credibility of the combined test and prediction of posttest odds of this screening method. It is most important to tell the patient(s) that this is a process of screening and not the final diagnosis. That can be given only on the basis of invasive intervention and defining of the fetal karyotype [18].

Table 5. Influence of the combined test on the pretest odds and probability of the outcome in relation to the likelihood ratio in case of positive and negative outcome

\begin{tabular}{|l|c|}
\hline Parameter & p Value \\
\hline Pretest odds/probability & 0.0040 \\
\hline Posttest odds/positive test & 0.3760 \\
\hline Posttest odds/negative test & 0.0002 \\
\hline Likelihood ratio/LR+ & 94.000 \\
\hline Likelihood ratio/LR- & 0.0600 \\
\hline Posttest probability/positive test & 0.2732 \\
\hline Posttest probability/negative test & 0.0002 \\
\hline
\end{tabular}

\section{CONCLUSIONS}

By examining the sensitivity and specificity of the combined screening test in the period of 11.$13^{+6}$ weeks gestation, we found that sensitivity of the test was 0.94 (94.0\%), and its specificity 0.99 $(99.0 \%)$. Pretest probability that the pregnant woman carries a fetus with a chromosomal abnormality is $1: 250$ or 0.004 . Posttest odds after the combined test to discover this abnormality was 0.3760 , and probability of the same case was 0.2732 if the test result was positive. Posttest odds after the combined test and the probability of the same case was identical if the test result was negative and amounted to 0.0002 .

\section{REFERENCES}

1. Ebrahim S, Daponte A, Guidozzi F. The impact of free antenatal care on perinatal mortality. Int J Gynaecol Obstet. 2000; 71(3): 205-207.

2. Alfirevic Z, Neilson JP. Antenatal screening for Down's syndrome. Br Med J. 2004; 329(7470): 811-812.

3. Spencer K. Age related detection and false positive rates when screening for Down's syndrome in the first trimester using fetal nuchal translucency and maternal serum $\beta$ hCG and PAPP-A. Br J Obstet Gynaecol. 2001; 108: 1043-1046.

4. Wald NJ, Watt HC, Hackshaw AK. Integrated screening for Down's syndrome on the basis of tests performed during the first and second trimesters. $\mathrm{N}$ Eng1 J Med. 1999; 341(7): 461-467.

5. Wald NJ, Rodeck C, Hackshaw AK, Walters J, Chitty L, Mackinson AM. First and second trimester antenatal screening for Down's syndrome: the results of the Serum, Urine and Ultrasound Screening Study (SURUSS). J Med Screen. 2003; 10(2): 56-104. (Erratum in: J Med Screen. 2006; 13(1): 51-52.)

6. Stojilkovic-Mikic T, Rodeck CH. Screening for chromosomal anomalies: first or second trimester, biochemical or ultrasound? Ann Acad Med Singapore. 2003; 32(4): 583-589.

7. Haddow JE, Palomaki GE, Knight GJ, Williams J, Miller WA, Johnson A. Screening of maternal serum for fetal Down's syndrome in the first trimester. N Engl J Med. 1998; 338(14): 955961.

8. Wald NJ, George L, Smith D, Densem JW, Petterson K. Serum screening for Down's syndrome between 8 and 14 weeks of pregnancy. International Prenatal Screening Research Group. Br J Obstet Gynaecol. 1996; 103(5): 407-412.

9. Snijders RJM, Farrias M, Kaisenberg C, Nicolaides KH. Fetal abnormalities. In: Sniijders RJM, Nicolaides KH, Eds. Ultrasound Markers for Fetal Chromosomal Defects. New York: Parthenon, 1996: 1-63.

10. Cunningham FG, Leveno KJ, Bloom SL, Hauth JC, Gilstrap L, Wenstrom KD, Eds. Prenatal diagnosis and fetal therapy. Williams Obstetrics. New York: McGraw-Hill, 2005: 313-340.

11. Wellesley D, Boyle T, Barber J, Howe DT. Retrospective audit of different antenatal screening 
policies for Down's syndrome in eight district general hospitals in one health region. $\mathrm{Br}$ Med $\mathrm{J}$. 2002; 325(7354): 15-17.

12. Lončar D, Varjačić M, Novaković T, Milovanović D, Janković S. Correlation between serum biochemical markers and early amniocentesis in diagnosis of congenital fetal anomalies. Bosnian J Basic Med Sci. 2010; 10(1): 9-14.

13. Hallahan T, Krantz D, Orlandi F, Rossi C, Curcio P, Macri S, Larsen J, Buchanan P, Macri J. First trimester biochemical screening for Down syndrome: free $\beta$-hCG versus intact hCG. Prenat Diagn. 2000; 20(10): 785-789.

14. Brigatti KW, Malone FD. First trimester screening for aneuploidy. Obstet Gynecol Clin North Am. 2004; 31(1): 1-20.

15. Nicolaides KH. Nuchal translucency and other first-trimester sonographic markers of chromosomal abnormalities. Am J Obstet Gynecol. 2004; 191(1): 45-67.

16. Spencer K, Souter V, Tul N, Snijders R, Nicolaides KH. A screening program for trisomy 21 at 10-14 weeks using fetal nuchal translucency, maternal serum free $\beta$-human chorionic gonadotropin and pregnancy-associated plasma protein-A. Ultrasound Obstet Gynecol. 1999; 13(4): 231-237.

17. Krantz DA, Hallahan TW, Orlandi F, Buchanan P, Larsen JW Jr, Macri JN. Firsttrimester Down syndrome screening using dried blood biochemistry and nuchal translucency. Obstet Gynecol. 2000; 96(2): 207-213.

18. Spencer K, Spencer CE, Power M, Moakes A, Nicolaides KH. One stop clinic for assessment of risk for fetal anomalies: a report of the first year of prospective screening for chromosomal anomalies in the first trimester. Br J Obstet Gynaecol. 2000; 107(10): 1271-1275. 